\title{
GIANT ACROCHORDON OF LABIA MAJORA - AN UNCOMMON MANIFESTATION OF A COMMON DISEASE
}

Gopikrishna Dharmapuri1 ${ }^{1}$ Grey Bernard Francis ${ }^{2}$, Arun Prasath Sivaraman ${ }^{3}$

${ }^{1}$ Assistant Professor, Department of General Surgery, Rajah Muthiah Medical College and Hospital, Annamalai Nagar, Chidambaram, Tamilnadu.

2Final Year Postgraduate, Department of General Surgery, Rajah Muthiah Medical College and Hospital, Annamalai Nagar, Chidambaram, Tamilnadu.

${ }^{3}$ Final Year Postgraduate, Department of General Surgery, Rajah Muthiah Medical College and Hospital, Annamalai Nagar, Chidambaram, Tamilnadu.

\section{ABSTRACT}

\section{BACKGROUND}

Acrochordon (Fibroepithelial polyp or skin tag) is a common benign neoplasm seen predominantly in obese individuals with an average of $46 \%$ incidence in the general population.[1] Measuring about 1 to $5 \mathrm{~mm}$ in size, they are usually located in the intertriginous areas. We report an unusual presentation of acrochordon.

\section{KEYWORDS}

Giant Acrochordon, Fibroepithelial Polyp, Labia Majora.

HOW TO CITE THIS ARTICLE: Dharmapuri G, Francis GB, Sivaraman AP. Giant acrochordon of labia majora - an uncommon manifestation of a common disease. J. Evolution Med. Dent. Sci. 2016;5(87):6521-6522, DOI: 10.14260/jemds/2016/1474

\section{BACKGROUND}

An acrochordon is a small, soft, common, benign, usually pedunculated neoplasm that is found particularly in persons who are obese. It is usually skin coloured or hyperpigmented and it may appear as surface nodules or papillomas on healthy skin. Most acrochordon vary in size from 2-5 $\mathrm{mm}$ in diameter, although larger acrochordon up to $5 \mathrm{~cm}$ in diameter. The most frequent localisations are neck and the axilla, but any skin fold including the groin may be affected.

\section{CASE REPORT}

A 26 years old woman presented with a mass hanging from left labia majora since 2 years. While cleaning her private parts, she first noticed a lemon sized mass which gradually increased in size. Patient experienced discomfort while walking due to the weight and rubbing of the mass between her thighs and became apprehensive of its growing size. There was no pain, pruritus, fever, redness, ulceration, bleeding, discharge, sudden increase in size, aggravating/relieving factors or diurnal change in size.

Patient was overweight (body mass index was 26.3 $\mathrm{kg} / \mathrm{m}^{2}$ ). Dermatological examination revealed a single, skincoloured, pedunculated, pear-shaped, non-tender, soft fleshy mass with wrinkled surface measuring $16 \times 9 \mathrm{~cm}$ and arising from a thin stalk from posterior part of left labia majora and distorting its shape. There was no redness, discharge or ulceration. The mass was non-pulsatile, non-reducible with no impulse on coughing, no palpable thrill or bruit with no regional lymphadenopathy. Systemic and genitourinary examination was normal. Blood sugar and lipid profile were normal. The mass was diagnosed as acrochordon due to its pedunculated attachment, soft consistency and free mobility.

Financial or Other, Competing Interest: None.

Submission 21-09-2016, Peer Review 18-10-2016,

Acceptance 25-10-2016, Published 29-10-2016.

Corresponding Author:

Dr. Grey Bernard Francis,

Room No. 301,

PG Doctors Men's Hostel,

RMMCH, Annamalai Nagar

Chidambaram-608002, Tamilnadu.

E-mail: greybernard@gmail.com

DOI: 10.14260/jemds/2016/1474

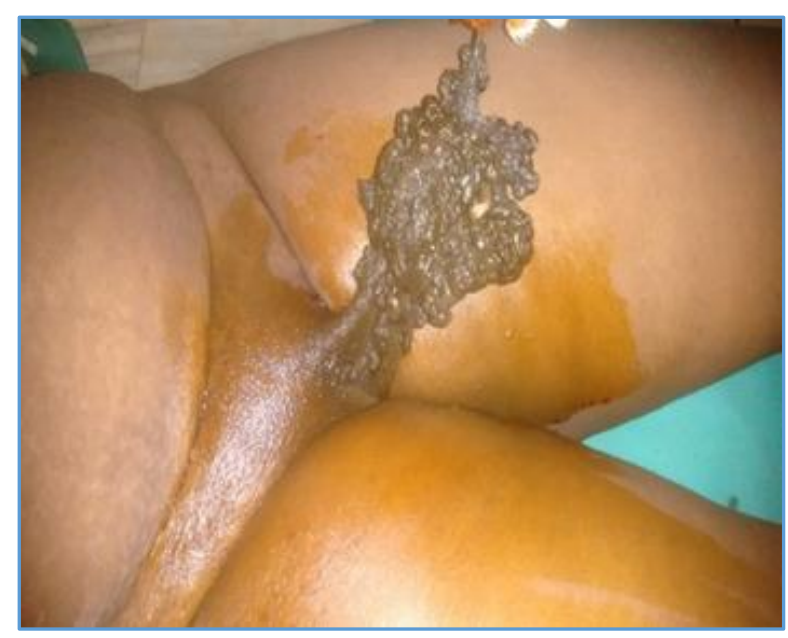

Figure 1. Giant Acrochordon of the Left Labia Majora: Pre-Operative Picture 2

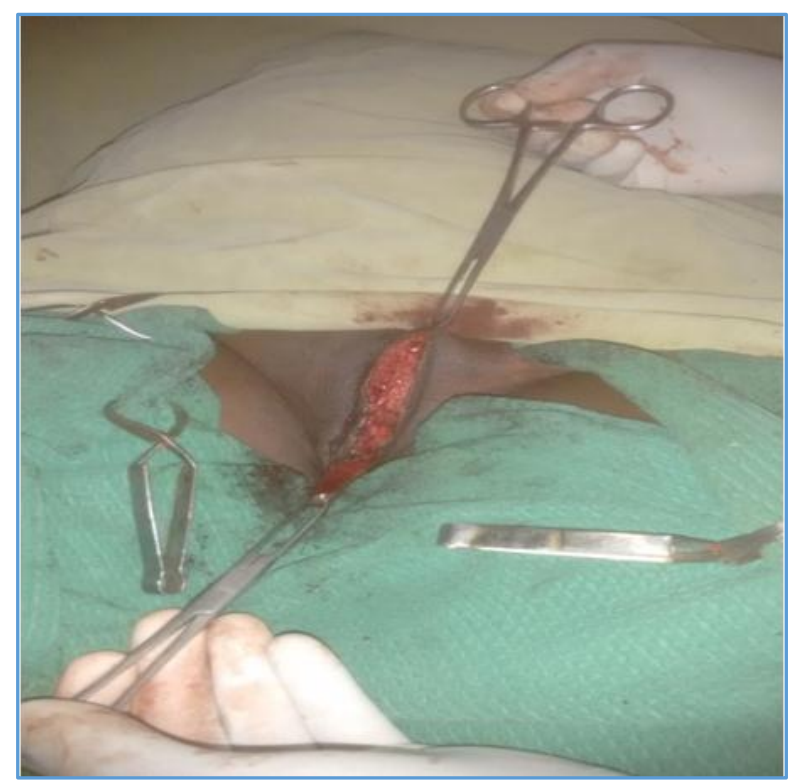

Figure 2. Intraoperative Picture 


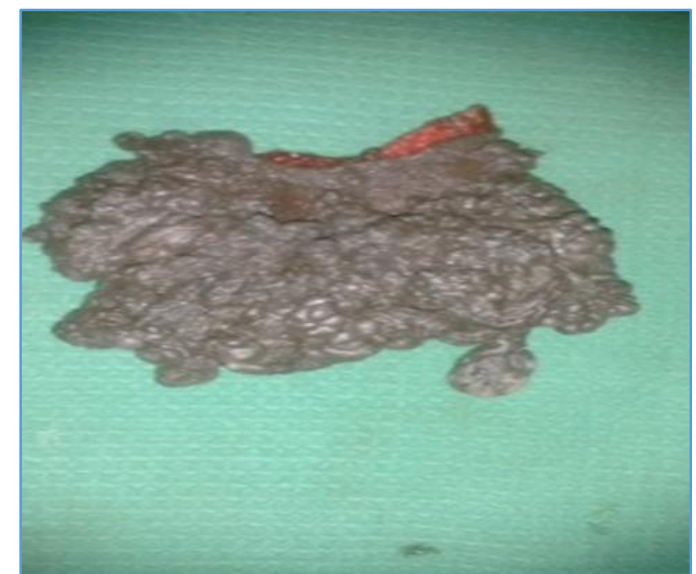

Figure 3: Length of the Excised Acrochordon

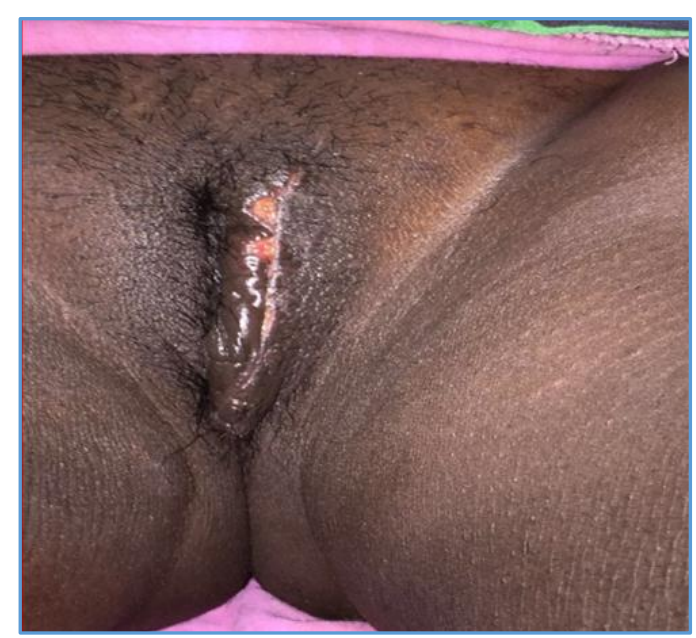

Figure 4. Post-Operative Picture

Giant acrochordon of the left labia majora (1 and 2) preoperative (3) Immediate post-operative (4) Length of the excised acrochordon (5) and 20 days after surgery (6) Mature stratified squamous epidermis with the underlying stroma showing increase in the fibrocollagenous tissue.

The lesion was elliptically excised. Histopathological examination revealed mature stratified squamous epithelium, increased fibrocollagenous tissue in the stroma, thickened blood vessels, stellate shaped fibroblast and sparse perivascular chronic mononuclear inflammatory infiltrate, squamous epidermis with the underlying stroma showing increase in fibrocollagenous; no evidence of malignancy.

\section{DISCUSSION}

In women, genital acrochordon is more common in the vagina than vulva and cervix ${ }^{[2]}$ with peak incidence at 20-40 years of age. It is rare in postmenopausal women. Large lesions may arise due to proliferation of mesenchymal cells within the hormonally sensitive subepithelial stromal layer of the lower genital tract. Acrochordons are associated with type 2 diabetes mellitus, insulin resistance, obesity, dyslipidaemias, pregnancy, genetic predisposition, human papilloma virus 6 and 11, acromegaly, Gardner syndrome, Birt-Hogg-Dube syndrome and Nonne-Milroy-Meige syndrome.[3]

The vulval acrochordons reported in the literature have ranged in size from 2.3 to $30 \mathrm{~cm} .{ }^{[4,5]}$ Ulceration, infection and inflammation can occur in giant acrochordons of vulva. Acrochordons rarely recur if not completely excised.[3]

\section{Differential Diagnosis}

The differential diagnosis of vulval acrochordon includes Hernia, site of presentation is internal ring of vulva. Cystic mass which may be reducible cough impulse present Hydrocele of canal of Nuck, Inguinal crease or Anterior labia majora. It can occur anywhere along inguinal canal. It is Cystic swelling, not reducible. Cough impulse negative. Transillumination positive. It is due to remnant of peritoneum.

Neurofibroma, a tumour formed on a nerve cell sheath, frequently symptomless but occasionally malignant lipoma. It is located in labia majora. It may be associated with other lipomas in abdomen or thigh. It is subcutaneous, soft fatty, non-cystic, asymptomatic and slow growing lesion.

Fibromas occur on the vulva as small exophytic projections Bartholin's cyst, Bartholin's gland is blocked and the gland becomes inflamed. Size ranges from that of a pea to that of an egg and form just within each side of the lower part of the opening of vagina vulvar varicosities, tend to occur most often during pregnancy. Haemangiomas, benign tumour consisting of the vascular epithelial cells. Most often this disorder affects new-born and infants under a year. Angioneurofibroma a rare tumour of vulva, a case report of a vulva angioneurofibroma in a Cameroonian women.

Hamartoma, lymphadenoma, angiomyofibroblastoma, cellular angiofibroma, sarcomas, angiomyxoma and dermatofibrosarcoma protuberans are other differential diagnosis.

Malignancy should be excluded in every case of fibroepithelial stromal polyp. Stellate and multinucleated stromal cells present near the epithelial-stromal interface are the most characteristic feature of acrochordon. Stromal cells may be positive for desmin, actin, vimentin, oestrogen and progesterone receptors. On the other hand sarcomas have identifiable lesion margins, homogeneous cellularity and lack the stellate and multinucleated stromal cells near the epithelial stromal interface.

\section{CONCLUSION}

We report an unusual presentation of a very common lesion presenting with large size, vulval location and appearance in postmenopausal overweight woman with no associated factors. Surgical excision with histopathological examination serves as diagnostic and therapeutic modality for such lesions.

\section{REFERENCES}

1. Ahmed S, Khan AK, Hasan M, et al. A huge acrochordon in labia majora- an unusual presentation. Bangladesh Med Res Council Bull 2011;37(3):110-1.

2. Dey M, Kumar R, Sriram R. Giant acrochordon of vulva. Med Sci (Turkey) 2014;3(2):1299-304.

3. Orosz Z, Lehoczky O, Szoke J, et al. Recurrent giant fibroepithelial stromal polyp of the vulva associated with congenital lymphedema. Gynecol Oncology 2005;98(1): 168-71.

4. Canalizo-Almeida S, Mercadillo-Pérez P, Tirado-Sánchez A. Giant skin tags: report of two cases. Dermatol Online J 2007;13(3):30.

5. Wani Y, Fujioka Y. A vulvar fibroepithelial stromal polyp appearing in infancy. Am J Dermatopathol 2009;31(5): 465-7. 